\title{
Near Field Scans of Tyre mounted Dipoles using a separate Phase Reference Antenna
}

\author{
Gregor Lasser* David Löschenbrand ${ }^{\dagger} \quad$ Christoph F. Mecklenbräuker $^{\dagger}$
}

\begin{abstract}
This paper reports on near field measurements of dipole antennas mounted inside a wheel for advanced tyre monitoring applications based on UHF RFID technology. We propose, implement and validate a near field measurement technique which avoids the need for any error prone feed cable. This technique is then used for measuring the patterns of two different dipole antennas attached to the inner surface of a tyre in four mounting configurations. Finally, the measured antenna patterns are discussed, as well as their implications for the channel characteristics when these dipoles are used.
\end{abstract}

\section{Introduction}

Advanced Tyre Monitoring Systems (ATMS) [1] are an enhancement of classical Tyre Pressure Monitoring Systems (TPMS) where sensors are employed directly into the tyre to measure vibration, temperature and pressure data. When this live data is available for the on board electronics, the effectiveness of several safety systems like anti-lock brake system (ABS) and Electronic Stability Program (ESP) is enhanced. For this data transfer an energy efficient transmission technique must be used, because the sensor units have to be extremely small, and therefore battery less, to enable integration into the tyre without mechanical problems. Previously, UHF Radio Frequency IDentification (RFID) was proposed [2] as a potential technology to power the sensor units and transfer data to the onboard electronics using backscattering technology. The channel measurements reported in [2] investigate the channel between a centrally mounted monopole antenna on the body floor pan of a vehicle and two different dipole antennas mounted on four positions on the interior sidewall of the tyre.

In this contribution we will present Near Field (NF) pattern measurements of the dipole antennas described in [2] mounted in a car tyre. The wheel rim, metal reinforcements in the tyre and its dielectric properties strongly influence the antenna pattern [3]. Measurements of such small antennas are delicate, and errors induced by the antenna feed cable are difficult to overcome [4-6]. We use a similar approach as described in $[4,6]$ using a small battery driven oscillator, but we perform NF measurements

\footnotetext{
*Vienna University of Technology, Institute of Telecommunications. email: gregor.lasser@tuwien.ac.at

$\dagger$ Christian Doppler Laboratory for Wireless Technologies for Sustainable Mobility, Vienna University of Technology
}

using a commercial spherical scanner system as described in the next section.

\section{Measurement System}

The measurement system used is a spherical theta over phi scanner. While the scan range in azimuth is unlimited $\left(0^{\circ} \leq \varphi \leq 360^{\circ}\right)$, the theta arm is limited to $-160^{\circ}<\theta<160^{\circ}$ by the phi stage. The standard measurement procedure requires a cable to be connected to the antenna, which then is routed through a rotary joint at the bottom of the phi stage and connects to a network analyzer. This method is prone to measurement errors if electrically small low gain antennas or symmetric antennas without proper baluns are measured, mostly because radiating sheath currents on the feed cable cannot be avoided.

Similar to $[4,6]$ we use a small battery driven oscillator with dimensions of $18 \mathrm{~mm} \times 9 \mathrm{~mm} \times 3 \mathrm{~mm}$ directly attached to the Antenna Under Test (AUT) to eliminate the feed cable. But in contrast to these citations we perform NF measurements on our AUTs. The benefits of NF scans of small AUTs when compared to conventional Far Field (FF) scans are faster scanning times for the same resolution and lower AUT positioning accuracy demands. Since phase information is essential for all NF measurements [7], a phase reference

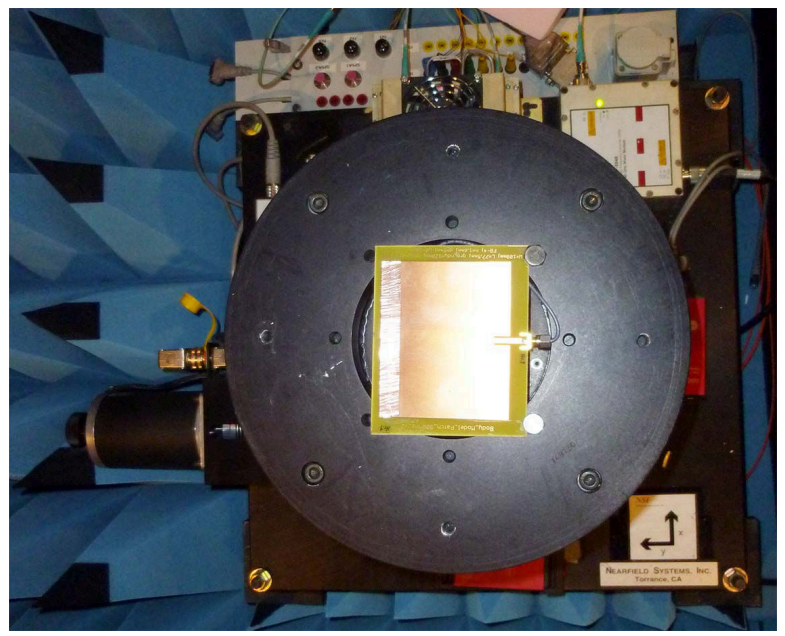

Figure 1: Phi Stage seen from top with mounted pickup antenna for NF phase reference. 
locked to the oscillator must be provided. For this purpose we use a pickup antenna mounted directly on top of the Phi stage, see Fig. 1. It is connected to the measurement hardware using the rotary joint of the phi stage. Since the pickup antenna is almost level with the metallic flange of the phi stage, we do not expect any measurable influence of the pickup antenna on the pattern of the AUT.

The assembly of the AUT and the oscillator is mounted on the phi stage using dielectric spacers. Therefore, the AUT rotates on the phi stage in full mechanical synchronization with the pickup antenna and the channel between AUT and pickup antenna remains unchanged throughout the measurement. The NF data is measured using a Vector Network Analyzer (VNA) with the pickup antenna directly connected to the input of receiver $R 1$, and the scanner probe antenna connected to the receiver $A$ input. The complex ratio $A / R 1$ is recorded and used to calculate the far field patterns using a commercial unmodified software.

\section{Measurement Results}

In this section we will present the measurement results obtained using the proposed measurement technique described before. All measurements were carried out at a frequency of $864 \mathrm{MHz}$. Prior to the actual measurements of the tyre mounted dipoles we performed validation measurements described below.

\subsection{Validation Measurements}

To validate our proposed measurement method we first measured a resonant dipole antenna manufactured from brass tube, see [6, Figure 3]. The small battery driven oscillator was attached to the dipole and it was mounted vertically in the chamber ontop of a dielectric support structure. To evaluate the effect of the oscillator circuit on the dipole pattern, three different measurements $(\mathrm{A}, \mathrm{B}, \mathrm{C})$ were conducted. additionally, the dipole was mounted horizontally on the dielectric support structure and again the pattern was measured. Fig. 2 shows a comparison of the three obtained pattern plots, where the coordinate system of the horizontal mounted dipole was converted to the one used for measurements A-C for comparison.

Measurement A shows an extremely frayed pattern which is explained by the fact the here by coincidence the dipole was mounted perfectly vertical above the pickup antenna, so that almost no power is captured from it. Additionally, the probe antenna used for the pattern measurements acts as a scatterer and provides a second path from the oscillator driven dipole to the pickup antenna. As

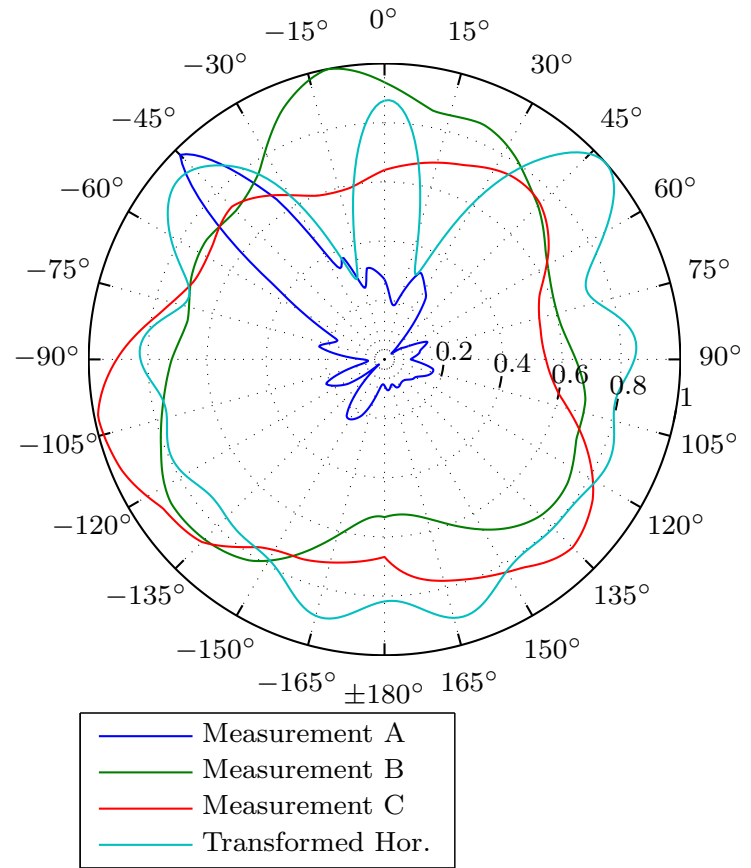

Figure 2: Measuered directional patterns of vertically mounted dipoles (A,B,C) and a horizontally mounted dipole transformed to the same coordinate system.

this path is now similar in power when compared to the direct path, the amplitude and phase of the reference signal depend on the theta arm position and the measurement results are corrupt. For measurements $\mathrm{B}$ and $\mathrm{C}$ there is a maximum derivation from maximum to minimum of $3 \mathrm{~dB}$, which is partially caused by the slight change in antenna geometry by the oscillator assembly. Measurement $\mathrm{C}$ was taken using the same setup as B but rotating the dipole by $90^{\circ}$ in azimuth. The corresponding plot in Fig. 2 really is very similar to the one of Measurement $\mathrm{B}$ if the rotation is considered. The transformed horizontal measurement shows even lower deviation from a perfect circle when a sector of $60^{\circ}$ centered at $\varphi=0^{\circ}$ is not considered. This sector is the one which was not captured by the theta arm because it corresponds to angles of $\theta>160^{\circ}$ in the initial measurement which are prohibited by the scanner geometry. Here some artefacts from the NF to FF transform are noticeable.

\subsection{Dipole in Tyre Measurements}

In this survey we measure the pattern of antenna configurations that were initially used for ATMS channel measurements, described in [2]. Four different mounting positions of dipole antennas on the sidewall inside a tyre are evaluated which are illustrated in [8, Fig. 2]: Parallel to the tread close to 


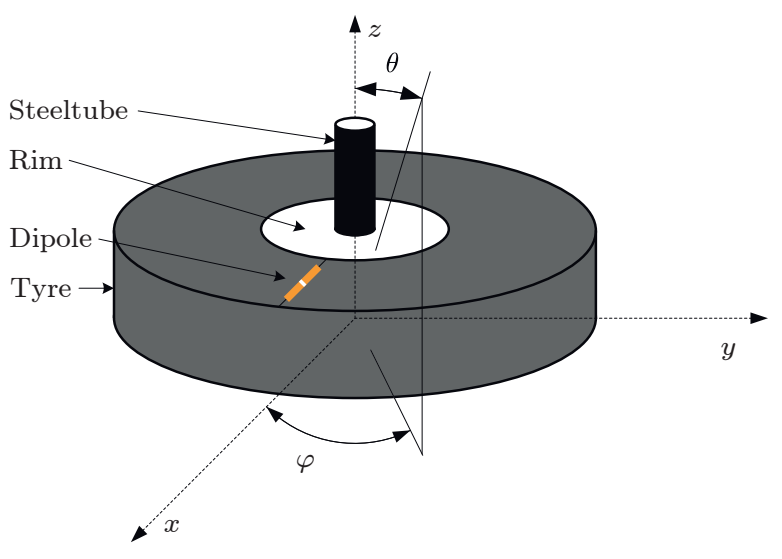

Figure 3: Schematic drawing of the tyre in the used coordinate system carrying a dipole mounted in the outer orthogonal position.

the car center (inner parallel), parallel to the tread but on the tyre sidewall facing away from the car (outer parallel) and orthogonal to the tread again for both sidewalls (inner orthogonal and outer orthogonal). For the used coordinate system and a dipole mounting example, see Fig. 3.

Two different dipole types manufactured of adhesive-coated copper tape are used: The long dipole $101 \mathrm{~mm} \times 10 \mathrm{~mm}$, and the short dipole $42 \mathrm{~mm} \times 10 \mathrm{~mm}$. The long dipole is resonant at the measurement frequency of $864 \mathrm{MHz}$ due to the dielectric loading of the tyre rubber. The oscillator assembly is placed on one arm of the dipole antenna, close to the feedpoint. Note that the used dipole antennas are wider than the oscillator assembly, so we do not expect a change of the antenna characteristics. For the orthogonal mounting positions only the short dipoles could be used due to mounting restrictions on the tyre sidewall.

Fig. 4 presents gain plots or the obtained measurements for outer mounting positions. As expected the patterns for the same orientations but different dipole lengths are very similar, both for the gain plot corresponding to electric field components in theta direction $E_{\theta}$ and for the gain corresponding to $E_{\varphi}$. The main beam is pointing towards low polar angles, and almost no power is radiated through the opposite tyre sidewall towards high polar angles. The behavior for the dipole mounted orthogonal to the tread is quite different: Here most radiation occurs at polar angles close to $\theta=90^{\circ}$, and the pattern is very frayed in azimuth. To make sure this effect is not an artifact due to dualpath propagation to the pickup antenna, as we saw for measurement A in Fig. 2 we also performed a FF measurement of this AUT and tyre mounting configuration using just the amplitude of the de- tected signal at the probe $|A|$. The results are very similar to the NF measurement so this behavior is real. One possible explanation is that in this orientation the dipole couples significantly with the two steel belt layers in the tread. The measured directivities for the outer mounting positions are $7.2 \mathrm{~dB}$ for both parallel mounted dipoles and $6.3 \mathrm{~dB}$ for the orthogonal dipole.

For the inner mounted dipoles the gain plots are shown in Fig. 5. The obtained plots are very similar to the ones for outer mounting positions if the polar axis is mirrored. Slight differences occur due to the asymmetrical rim, and the additional steel tube at the outer position used for the initial channel measurements [2]. Measured directivities are $7.3 \mathrm{~dB}$ for both parallel mounted dipoles and $5.9 \mathrm{~dB}$ for the orthogonal dipole.

Although the free space antenna measurements conducted here do not directly relate to the situation when the wheel is mounted inside the wheelhouse of a car with less then one wavelength separation, the fundamental properties of the radiation patterns observed here have a strong influence on the measured channel described in [2]. The frayed behavior of the orthogonal mounted dipoles patterns will result in more multipath components in the channel to the onboard unit antenna, which results in emphasis on higher frequency spectral components of the channel coefficients, as illustrated in [2, Fig. $5 \mathrm{~b}$ and Fig. 9b].

\section{Conclusion}

In this contribution we present and validate a $\mathrm{NF}$ measurement method to capture radiation patterns of small dipole antennas mounted inside a vehicular wheel. As long as the power scattered from the probe is small when compared to the power directly reaching the pickup antenna from the AUT, accurate NF measurements of arbitrary AUTs are obtained without the negative side effects of a cable feed. For parallel to the tread mounted dipoles the radiated power is concentrated on the side of the tyre where the dipole is mounted and otherwise the pattern is similar to the one of a pure dipole. The orthogonal mounted dipole shows a very frayed pattern in azimuth and most radiation occurs in direction of the tread. These pattern differences relate to the different measured channel characteristics presented in [2].

\section{Acknowledgement}

This work has been funded by the Christian Doppler Laboratory for Wireless Technologies for Sustainable Mobility. The financial support by the Federal Ministry of Economy, Family and Youth and the National Foundation for Research, Technology and Development is gratefully acknowledged. 

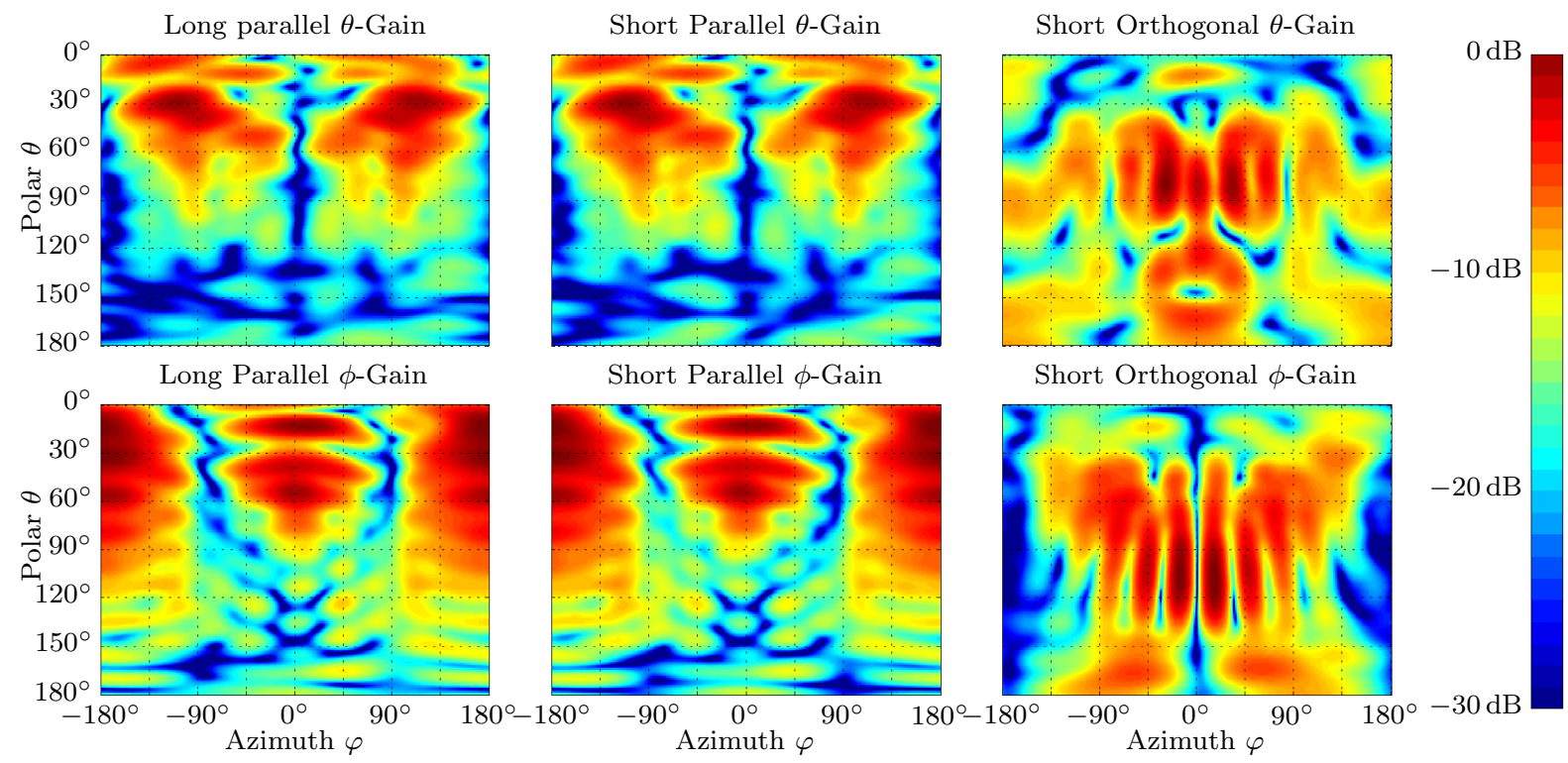

Short Orthogonal $\phi$-Gain

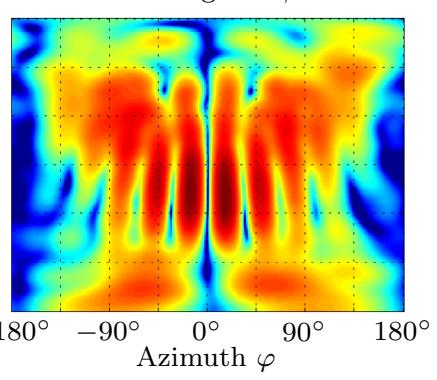

.
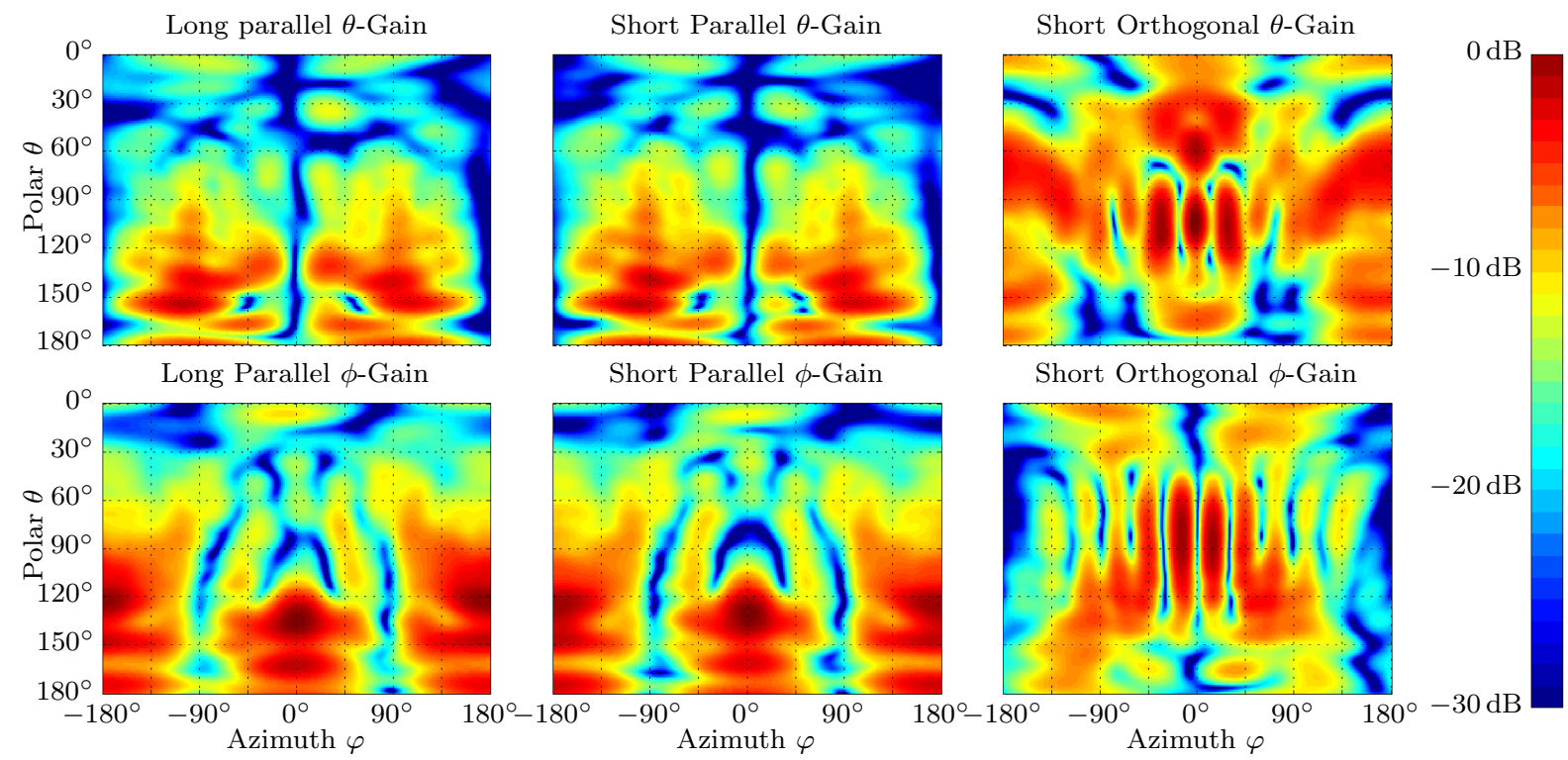

Figure 5: Normalized measured gain patterns for dipoles mounted on the inner position.

\section{References}

[1] S. C. Ergen, A. Sangiovanni-Vincentelli, X. Sun, R. Tebano, S. Alalusi, G. Audisio, and M. Sabatini, "The tire as an intelligent sensor," IEEE Trans. Comput.-Aided Design Integr. Circuits Syst., vol. 28, pp. 941-955, Jul. 2009.

[2] G. Lasser and C. F. Mecklenbräuker, "Dual-band channel measurements for an advanced tyre monitoring system," $\mathrm{Ve}$ hicular Technology Conference. VTC 2010-Spring. IEEE 71th, pp. 1-5, May 2010.

[3] J. Grosinger, G. Lasser, C. F. Mecklenbräuker, and A. L. Scholtz, "Gain and efficiency measurement of antennas for an advanced tire monitoring system," in Antennas and Propagation Society International Symposium (APSURSI), 2010 IEEE, Jul. 2010, pp. 1-4.

[4] A. K. Skrivervik and J.-F. Zürcher, "Distortions of measured antenna patterns caused by dielectric support structures," AUTOMATIKA - Journal for Control, Measurement,
Electronics, Computing and Communications, vol. 43, no. $1-2$, pp. $55-61$, Oct. 2002.

[5] T. Fukasawa, K. Shimomura, and M. Ohtsuka, "Accurate measurement method using fiber-optics for an antenna on a portable telephone," in Wireless Communication Technology, 2003. IEEE Topical Conference on, Oct. 2003, pp. 138-139.

[6] L. W. Mayer and A. L. Scholtz, "Gain and input impedance measurement for UHF transponder antennas," 2008 International Symposium on Antennas and Propagation, p. 4, 2008.

[7] J. Hansen, Ed., Spherical Near-Field Antenna Measurements, ser. IET Electromagnetic Wave Series 26. London, UK: The Institution of Engineering and Technology, 2008.

[8] G. Lasser, R. Langwieser, F. Xaver, and C. F. Mecklenbräuker, "Dual-band channel gain statistics for dualantenna tyre pressure monitoring RFID tags," in Proceedings of the 2011 IEEE International Conference on RFID. Orlando, Fl: IEEE, Apr. 2011, pp. 57-61. 